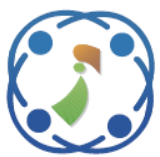

\title{
Autonomous Distributed Power Control in Multi-Channel Cognitive Femtocell Network: Feasibility and Convergence
}

\author{
Anggun Fitrian Isnawati ${ }^{1,2 *}$ \\ Risanuri Hidayat ${ }^{1}$ \\ Selo Sulistyo ${ }^{1}$ \\ I Wayan Mustika ${ }^{1}$ \\ ${ }^{I}$ Department of Electrical Engineering and Information Technology, Faculty of Engineering, \\ Universitas Gadjah Mada, Yogyakarta, Indonesia \\ ${ }^{2}$ Sekolah Tinggi Teknologi Telematika Telkom, Purwokerto, Indonesia \\ *Corresponding author’s Email: anggun.s3te13@mail.ugm.ac.id, anggun@ st3telkom.ac.id
}

\begin{abstract}
Dynamic user in mobile communication encourages the implementation of self-organized and noncooperative distributed power control. To be implemented, the power control must meet the feasible and convergent conditions. Feasibility of power control must be qualified for non-negative power vectors and the limit of maximum power, whereas convergence is tested by the speed for achieving of convergent conditions on fixed point. If it is feasible, then the system will be convergent, but when it is infeasible then the power transmit of user will be negative and the system is never reach the convergence. While the semi feasible condition requires the implementation of a proposed method of HDCPC. When power transmit of user exceeded the maximum power, $\mathrm{P}_{\max }$, HDCPC method choose the transmit power that equal with $1 / 2 \mathrm{P}_{\max }$ compared to use $\mathrm{P}_{\max }$ that required on DCPC or to force the user turned off the power transmit on GDCPC. Results showed that it would be more efficient in power usage than DCPC and more implementable than GDCPC. Proposed HDCPC is done when there is no option of handover channel, while the results after user move to another channel is able to achieve the SINR target and spent less power. Related to the convergence analysis, it can be concluded that the larger the SINR target, the longer iterations required to achieve a convergent condition.
\end{abstract}

Keywords: Feasibility, Convergence, Distributed power control, Non-negative power vector, HDCPC.

\section{Introduction}

Co-channel interference caused by frequency reuse is a factor that mostly limits the system capacity in the high capacity cellular communication. Therefore a power control scheme is required to control the interference of channel allocation. The scheme aims to adjust the transmitter power in each link so that it can minimize interference to other receiver [1].

There are two categories in the power control for cognitive radio networks: centralized power control (CPC) and distributed power control (DPC). CPC algorithm optimizes the power allocation to achieve the equal SIR (signal to interference ratio) in all radio links, and the optimal solution for the $\mathrm{CPC}$ has been proposed by Zander [2]. In CPC, all information necessary to manage the network must be known in the central station and needs the high power consumption in user side. CPC requires all users to have knowledge of all radio links in the network, which is computationally very expensive and impossible to be implemented in real time for any large systems.

To reduce the complexity of the $\mathrm{CPC}$, a method of power control compatible to the cognitive radio systems with self-organized user characteristics which uses the approach of autonomous distributed power control, is required. For DPC, each user controls the power transmission only using local information. A comparison between CPC and DPC in feasibility and convergence has been investigated by [3].

The need of power control that is implementable for a dynamic and distributed user is able to control 
the power independently to make it more flexible. Designing an efficient power control scheme will be very challenging especially in femtocell, mainly because of the nature of the autonomous network [4]. Power control schemes are studied mainly to minimize interference and ensure the fulfillment of quality of service (QoS) [5], to maximize the capacity and to reduce the energy consumption [6], to realize the green wireless communication and to overcome the trade-off QoS with efficiency energy better [7] and more effectively to overcome the noise [8][9].

The importance of testing the feasibility is whether a system or method is implemented in practice. Feasibility in terms of power control shown by the value of the power generated from a method/ scheme must be non-negative power vector [10] meaning that the power is positive and implementable to reach a specified SINR target with the minimum power consumption. When the system is feasible, then all users can be supported, which means that the transmit power control (TPC) produces the zero outage on a minimum transmit power consumption. In the infeasible systems, some users are unlikely to achieve the SINR target. In some users, because the TPC mechanism does not consider the feasibility, then the user will use a maximum power although not able to achieve the SIR target. This poses interference to other users and expands the number of non-supported user [11].

Beside test on feasibility, testing on convergence of power control scheme is also important. Commonly, the computational complexity will affect the speed of convergence. The unique convergence towards one particular point can occur on the systems that are feasible or not. If conditions are infeasible many users do not meet the SINR target despite using maximum power. It will later generate an outage ratio and higher power consumption [12]. But, if the algorithm converges, it reduces the outage probability and the total consumed power and its convergence as a consequence cannot be guaranteed in an infeasible system [11].

The importance of the feasibility and convergence check has been discussed in several papers, including for Cognitive Radio [13], Centralized [14], Distributed [15], Game Theory Power Control [16], Distributed Constrained Power Control [17] Generalized Distributed Constrained Power Control [18], in Femtocell [19] or in Macrocell [20], with Single channel or Multi channel [10].

However, these studies mostly have a power control algorithm that only concerns with feasibility and convergence. Feasibility in this case related to the power update of the user along with the achievement of a specified SINR target and the number of channels allocation. It also includes the convergence of user power usage and achievement of the SINR target by the user. Convergence in this study included the analysis of the system speed in achieving converges with the change of SINR target. Moreover, this study is related to the research sustainability from Sooyeol [21], which discussed two methods namely DCPC [17] and GDCPC [18]. In Sooyeol [21] has not discussed the comparison of the two methods in terms of feasibility in transmit power of user, the achievement of the SINR target and the convergence rate. However, each of these methods has disadvantages in terms of transmit power efficiency namely the use of maximum power at DCPC and forcing the user not to transmit at GDCPC. Therefore, in this proposed model, we discussed about the solution of the existing disadvantage in those two previous methods through the proposed HDCPC using transmit power $p_{i}^{*}=\frac{1}{2} P_{\max }$ were still able to achieve sufficient SINR (tolerable SINR). This method is performed when the user exceeds $P_{\max }$ so it does not harm the user or another user. In addition, this study also tested the feasibility and convergence based on user behavior in a multi-channel femtocell network.

The organization of this paper is presented as follows. The system model is described in Section II, and Section III explains the feasibility and convergence of autonomous distributed power control in femtocell network. Section IV presents the simulation results, and conclusions are given in Section V.

\section{System Model}

The system model of cognitive femtocell network in this study is shown in Fig. 1. This study used the femtocell users who used multiple channels simultaneously.

In this experiment, each femtocell consisted of multiple users. Every user in a one femtocell could not use the same channel, thus the same channel usage was only be for the user in a different femtocell. The components of cognitive femtocell network system as shown in Fig. 1 included femtocell user equipment (FUE) as a secondary-user transmitter and femtocell access point (FAP) as a secondary-user receiver.

Figure 2 shows the channel division scheme in femtocell users link gain matrix $\mathrm{H}$ that can be determined based on these users. As a multi-channel, it was necessary to check matrix $\boldsymbol{H}$ in each channel (user group). 


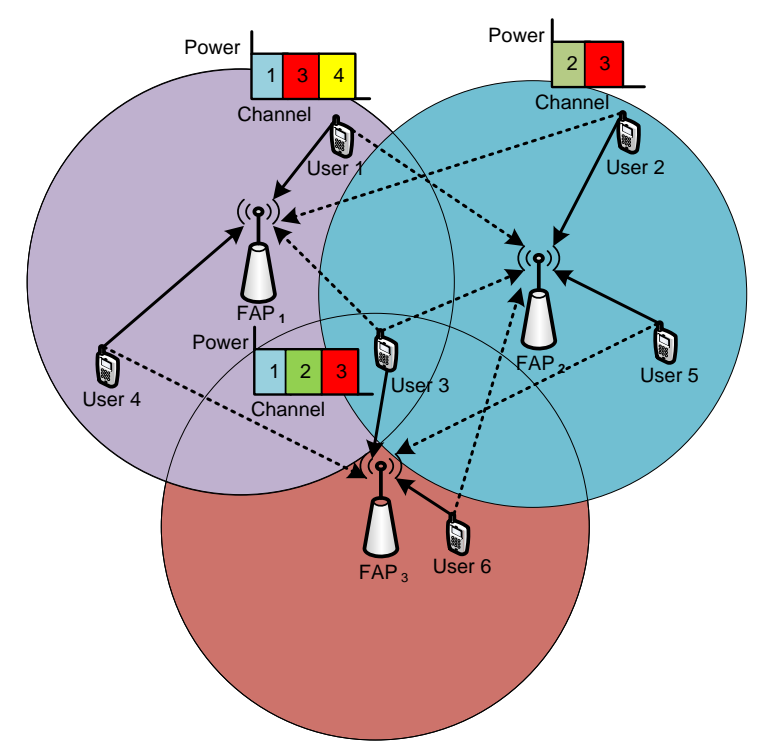

Figure.1 Cognitive femtocell network topology

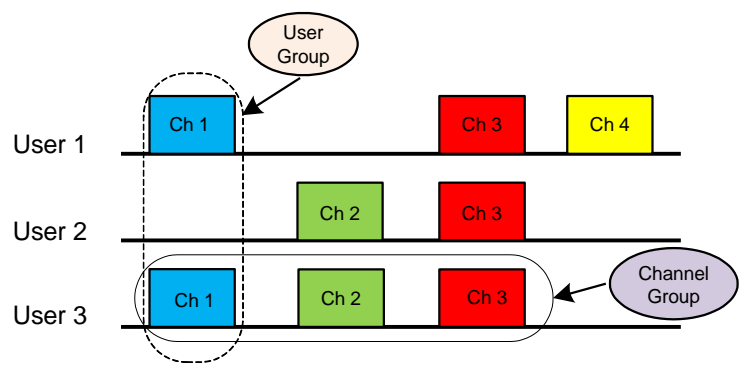

Figure.2 Channel division scheme in femtocell users

The matrix $\boldsymbol{H}$ was formed by link gain among users using the same channel, as shown in Fig.2 on the channel sharing scheme of femtocell user. Based on the feasible solution equation of user group, the form of the matrix $\boldsymbol{H}$ has been obtained as follows:

$$
\begin{array}{ll}
\boldsymbol{H}_{1}=\left[\begin{array}{ll}
\mathrm{h}_{11} & \mathrm{~h}_{13} \\
\mathrm{~h}_{31} & \mathrm{~h}_{33}
\end{array}\right] & \boldsymbol{H}_{2}=\left[\begin{array}{ll}
\mathrm{h}_{22} & \mathrm{~h}_{23} \\
\mathrm{~h}_{32} & \mathrm{~h}_{33}
\end{array}\right] \\
\boldsymbol{H}_{3}=\left[\begin{array}{lll}
\mathrm{h}_{11} & \mathrm{~h}_{12} & \mathrm{~h}_{13} \\
\mathrm{~h}_{21} & \mathrm{~h}_{22} & \mathrm{~h}_{23} \\
\mathrm{~h}_{31} & \mathrm{~h}_{32} & \mathrm{~h}_{33}
\end{array}\right] & \boldsymbol{H}_{\mathbf{4}}=\left[\mathrm{h}_{11}\right]
\end{array}
$$

$\boldsymbol{H}_{\boldsymbol{k}}=\left(h_{i j}\right)$ is normalized link gain matrix in channel $k$ that fulfill this equation:

$h_{i j}=\gamma_{i}^{\operatorname{tar}}\left(\frac{G_{i j}}{G_{i i}}\right)$ for $\mathrm{i} \neq \mathrm{j} ; \mathrm{h}_{\mathrm{ij}}=0$ for $\mathrm{i}$

with $\gamma_{i}{ }^{t a r}$ is SINR target. The link gain between the user $i$ as transmitter (Tx) and the user $i$ as receiver $(\mathrm{Rx})$ is denoted by $G_{i i}$. The gain of the user $j$ on the link between the $\mathrm{Tx}$ user $j$ and the $\mathrm{Rx}$ user $i$ is denoted by $G_{i j}$.

\section{Feasibility and Convergence of Autonomous Distributed Power Control in Femtocell Network}

\subsection{Feasibility of Power Control}

Feasibility in the autonomous distributed power control means that all initial power controls are able to achieve the conditions converges at a certain power value $\left(P^{*}\right)$ and at the same time all users reach the SINR target on the positive power value up to the value of maximum power [22]. By testing the feasibility, it can be identified that if user power has a negative value means it is impossible to achieve the desired SINR target. Feasibility can also be seen by the user power usage. Because when the user power exceeds $P_{\max }$ value, the condition is referred to as an infeasible condition, even though the SINR target can be achieved.

From these discussions it can be taken feasibility limits on DPC given to every user, if applied to a single channel [14]:

$P^{*}=(I-H)^{-1} \eta$

with $P^{*}$ is total power of user in Watts and $\boldsymbol{H}$ is link gain matrix. When it applied to the multiple channels, it must be based on the following power value [10]:

$P(i)=\sum_{k=1}^{K} P_{k}(i)=\sum_{k=1}^{K}\left(\left(I-H_{k}(i) \cdot \delta_{k_{i} k_{j}}\right)^{-1} \cdot \eta_{k}(i)\right) i \leq N$

with $\mathrm{K}$ is total number of channel, $k$ is channel number, and $\delta_{k_{i} k_{j}}$ is used to show whether two users used the same channel or not. If the value of index $i$ and $j$ are the same, or in another words the both users use the same channel then $\delta_{k_{i} k_{j}}=1$. If they are not in the same channel then $\delta_{k_{i} k_{j}}=0$.

By considering the factors of interference, it is necessary to limit the maximum power that is:

$p_{\min } \leq p_{i}^{*} \leq P_{\max }$

with $p_{\min }=0 \mathrm{~W}$ or the value of specific user power and $p_{i}^{*}$ is chosen power of user in Watts.

The determination of new user power in the process of power update is associated with previous user power on DPC approach or also called Power Balancing Algorithm (PBA) [17]:

$p_{i}^{(t+1)}={\frac{\gamma_{i}{ }^{t a r}}{\gamma_{i}^{(t)}}}_{{ }^{(t)}}^{(t)}$

with $p_{i}{ }^{(t+1)}$ is the next power transmit, $p_{i}{ }^{(t)}$ is current power of user $i$, and $\gamma_{i}{ }^{(t)}$ is achieved SINR of user $i$.

Then, the concept of power update is based on the maximum power constraint and developed into two types: Distributed Constrained Power Control (DCPC) and Generalized Distributed Constrained Power Control (GDCPC) by the following formula. 
DCPC [17]:

$p_{i}^{(t+1)}= \begin{cases}\frac{\gamma_{i}{ }^{\text {tar }}}{\gamma_{i}{ }^{(t)}} p_{i}^{(t)}, & \text { if } \frac{\gamma_{i}{ }^{\text {tar }}}{\gamma_{i}^{(t)}} p_{i}^{(t)} \leq P_{\max } \\ P_{\text {max }}, & \text { if } \frac{\gamma_{i}{ }^{\text {tar }}}{\gamma_{i}^{(t)}} p_{i}^{(t)}>P_{\text {max }}\end{cases}$

with $p_{i}^{*}=P_{\max }$ or transmits in the maximum power. GDCPC [18]:

$p_{i}^{(t+1)}= \begin{cases}\frac{\gamma_{i}{ }^{\text {tar }}}{\gamma_{i}{ }^{(t)}} p_{i}^{(t)}, & \text { if } \frac{\gamma_{i}^{\text {tar }}}{\gamma_{i}^{(t)}} p_{i}^{(t)} \leq P_{\text {max }} \\ 0, & \text { if } \frac{\gamma_{i}^{\text {tar }}}{\gamma_{i}^{(t)}} p_{i}^{(t)}>P_{\text {max }}\end{cases}$

with $p_{i}^{*}=0$ or user doesn't transmit.

In this study, a proposed formula of Half DCPC (HDCPC) was different from the two formulas as shown as follows:

$p_{i}^{(t+1)}= \begin{cases}\frac{\gamma_{i}{ }^{\text {tar }}}{\gamma_{i}{ }^{(t)}} p_{i}^{(t)}, & \text { if } \frac{\gamma_{i}^{\text {tar }}}{\gamma_{i}{ }^{(t)}} p_{i}^{(t)} \leq P_{\text {max }} \\ \frac{1}{2} P_{\text {max }}, & \text { if } \frac{\gamma_{i}^{\text {tar }}}{\gamma_{i}^{(t)}} p_{i}^{(t)}>P_{\text {max }}\end{cases}$

with $p_{i}^{*}=\frac{1}{2} P_{\max }$ or user transmits with half of the maximum power.

The reasons for using $\mathrm{HDCPC}$ with $p_{i}^{*}=\frac{1}{2} P_{\max }$ was because the user power transmit in DCPC [17] was too high if each user always used a maximum power, so beside it could cause the large interference to other users, it would also result in power waste of devices although SINR target could not be achieved. While in GDCPC [18], it would be very impossible in its implementation to force the user not to transmit and succumb to the benefit of other users. This was due to the selfish nature of every user. Therefore, we have made a value in the middle of these two values with a view of making the user still able to transmit and communicate with other users but in the power that was not wasteful as the maximum power usage.

By using the feasible solution of power control as in equation (2), then the power vector for all $k$ user group can be defined as follows [14]:

$P_{k}^{*}=\left(I-H_{k}\right)^{-1} \eta_{k}$

with $P_{k}^{*}$ is power vector for user group that uses channel $k$ and $\eta_{\mathrm{k}}=\left(\eta_{\mathrm{i}}\right)$ is normalized noise vector. The number of elements of $P_{k}^{*}$ is the same with number of user using the same channel.

The requirement of system would be feasible if:

1. The value of $P^{*}$ is a non-negative power vector [23]

2. The |eigenvalues of $\boldsymbol{H} \mid<1$ [24]

Based on [10], existence and feasibility have the same meaning, so if it exists, the system will be feasible to be implemented. Feasibility is also associated with the maximum power, $P_{\max }$. If these two conditions are met but the power used exceeds the maximum power it will not be feasible. It is also the underlying principle of DCPC and GDCPC. To maintain feasibility, it can be done in various ways, including SU removal [12], to make a lower interference by widening the distance between the users and decreasing the SINR target.

\subsection{Convergence of Iterative Power Control}

Because it is associated with the iteration algorithm, it is necessary to note in the DPC scheme to prove the convergence. The requirements of convergence have to meet some of the properties: Positivity, Monotonicity and Scalability [25].

Convergence and uniqueness have the same meaning, so that if the iteration system is convergent, it will reach certain unique point [26]. The characteristic of the convergence of the algorithm with full information can be applied to the distributed systems [27]. The system is called convergent if during the process of power update no more changes in power, or a very little power changes $(\varepsilon)$ [28].

\section{Simulation Results}

Based on simulation result, the feasibility and convergence test was done on three types of DPC: DCPC [17], GDCPC [18] and the proposed HDCPC, as the sustainability of the research by Sooyeol [21]. This is because in Sooyeol [21] not discuss the feasibility and convergence system based on iterative methods, but only discusses the influence of the distance to interference and also the effect of distance on the number of supported users that are still able to be handled by the system.

At the initial stage, the feasibility and convergence test was performed on the DPC method or often referred to Power Balancing Algorithm (PBA) as outlined in equation (6). The feasibility of a system can be affected by a range of users, number of users and the SINR target. When the system feasible, the farther the user from the receiver, the greater the transmitted power.

Based on the feasibility, the DPC system is divided into three conditions: 1) infeasible condition, 2) semi feasible condition, and 3) ideal feasible condition. As for the analysis of convergence, DPC system will be analyzed based on the speed in achieving convergent.

\subsection{Infeasible Condition}

Based on the system model, link gain matrix $\boldsymbol{H}$ of the user group as in a system model can be seen as:

$$
\boldsymbol{H}_{\mathbf{1}}=\left[\begin{array}{cc}
0 & 1.523757013 \\
0.180668734 & 0
\end{array}\right]
$$




$$
\begin{aligned}
\boldsymbol{H}_{2} & =\left[\begin{array}{ccc}
0 & 2.945042026 \\
0.237085318 & 0
\end{array}\right] \\
\boldsymbol{H}_{3} & =\left[\begin{array}{ccc}
0 & 0.746083662 & 1.523757013 \\
4.840239828 & 0 & 2.945042026 \\
0.180668734 & 0.237085318 & 0
\end{array}\right] \\
\boldsymbol{H}_{4} & =[0]
\end{aligned}
$$

The test results of eigenvalue of $\boldsymbol{H}$ are shown as follows:

$$
\begin{aligned}
& \text { eig } H_{1}=\left[\begin{array}{r}
0.524685859 \\
-0.524685859
\end{array}\right] \text { eig } H_{2}=\left[\begin{array}{r}
0.835599321 \\
-0.835599321
\end{array}\right] \\
& \text { eig } H_{3}=\left[\begin{array}{r}
2.345132222 \\
-1.850807570 \\
-0.494324652
\end{array}\right] \text { eig } H_{4}=[0]
\end{aligned}
$$

It can be shown that some eigenvalues of matrix $\boldsymbol{H}$ met the qualify leigenvalue of $H \mid<1$, but there were some of them not qualified. This was because of the use of channels simultaneously which were most numerous in channel 3 of matrix $\boldsymbol{H}_{\mathbf{3}}$, so that the user group was not feasible. Associated with eigenvalues not qualified, then the power vector results would also be affected. Nevertheless, there was one user in the group channel 3 whose value was still eligible with the value of |eigenvalue of $\boldsymbol{H} \mid<1$, as shown in the user 3 channel $3,|-0.494324652|$, so that the results of the power vector $P_{3}$ is in positive value.

The feasible solution of power vector refers to the feasibility test in centralized power control as a reference to the distributed iterative power control:

$$
\begin{array}{rlrl}
P_{1} & =\left[\begin{array}{l}
0.151698 \\
0.901663
\end{array}\right] \mathrm{mW} & P_{2}=\left[\begin{array}{l}
0.662611 \\
0.219854
\end{array}\right] \mathrm{mW} \\
P_{3}=\left[\begin{array}{c}
-0.0444302 \\
-0.128357 \\
0.0243005
\end{array}\right] \mathrm{mW} & P_{4}=[0.0143068] \mathrm{mW}
\end{array}
$$

The results of the vector power were in accordance with the results of |eigenvalue matrix $\boldsymbol{H} \mid$ before and it can be shown that in $P_{3}$ had an infeasible result, as eigenvalue of $\boldsymbol{H}_{3} \mid$. This was because the value of the power vector was negative. So if the iteration process was carried out as the process of power update, it would generate a value undefined. Infeasible condition usually occurs because the distance between the users and the FAP is too far or the distance among the users is too close causing high interference. However, not all users in the group $P_{3}$ had negative power vector. User 3 channel 3 at $P_{3}$ had a positive value equal to $0.0243005 \mathrm{~mW}$. This was because the value of |eigenvalue of $\boldsymbol{H} \mid<1$ meaning the eligible for the feasible conditions.

\subsection{Semi Feasible Condition}

The results of the feasible solution can be used as a reference for the iteration process on distributed power control and the results shown in Fig. 3 and 4.

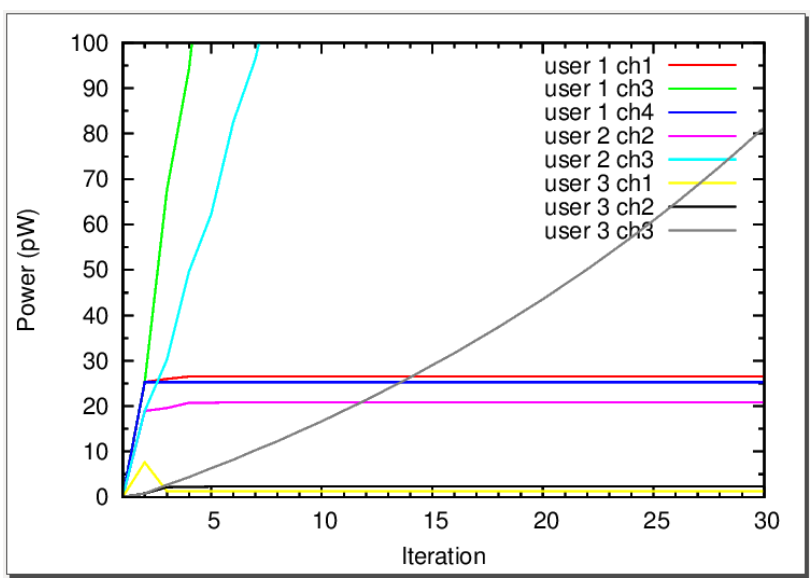

Figure.3 Power update in semi feasible condition

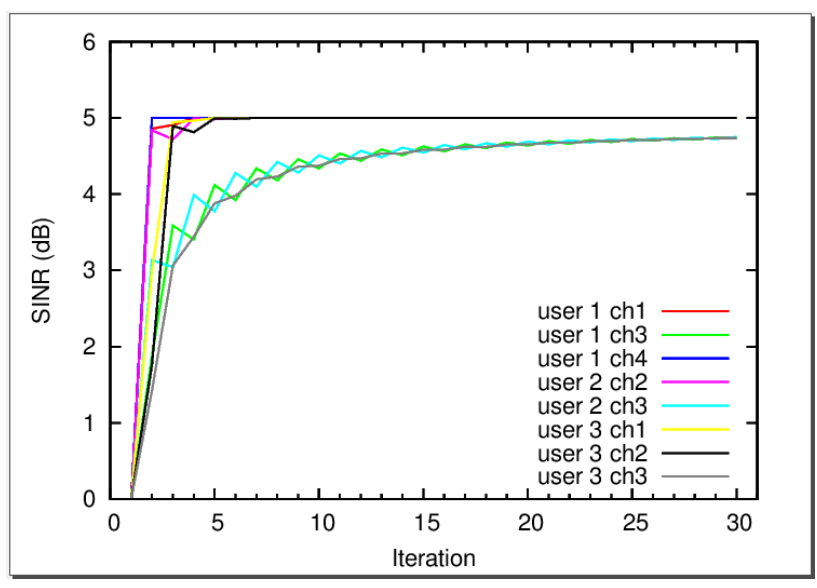

Figure.4 SINR user in semi feasible condition

Based on the results in Fig. 3 it can be concluded that when system was feasible, the iteration process of achieving convergence was very short, while for condition that is infeasible then the iteration would be very long (divergent) and power consumption exceeds the maximum power $\left(P_{\max }\right)$. The more users who use the same channel simultaneously, the longer iterations are required to achieve the convergence. This can be seen in Fig. 3 for the use of channel 3.

In the same condition, the more the users using the same channel simultaneously, the greater the power transmitted by the user when reaching the convergence. This can be seen in Fig. 3 for the use of channel 3. In this figure, it appears that the more users who used the channel simultaneously, the higher power required, even exceeding the maximum power. The convergence of the power of the users who used this channel as also the longer reached, could be up to hundreds or even thousands of iterations with the use of power greater than $P_{\text {max }}$.

Figure 4 showed that the more users who use the same channel simultaneously, the longer the convergence to be achieved. It was presented by users who used channel 3 that has reached 
convergence in 20th iteration. Despite achieving convergence condition, but this user was only able to achieve SINR of $4.8 \mathrm{~dB}$, meaning unable to achieve the SINR target. It was unequal in the case with users who used channel 1 , channel 2 , and channel 4 that were able to achieve the SINR target of $5 \mathrm{~dB}$.

DCPC, GDCPC and HDCPC methods were applied to the conditions of semi feasible where the treatment was done in power value that exceeded the maximum power, $\mathrm{P}_{\max }$. Treatment was performed on the user experience of semi feasible condition that could turn into ideal feasible or at least there was an increase in SINR performance in terms of power usage. The treatment could be done in two ways: 1) handover-channel based and 2) fixedchannel based.

\subsubsection{Handover-channel Based Treatment}

If the condition was still possible to move the channel, it was done by moving the user who originally used the full-channel to the emptychannel (handover channel). For this case, the channel handover occurred at the user 2 which initially used channel 3 and then moved to channel 4 . This was because the channel 3 is a full-channel (poor channel quality), while the channel 4 was channel with a few users (good channel quality).

Based on Table 1, it can be concluded that the process of channel handover can improve the user SINR so as to achieve the SINR target. It can be seen in all users who used channel 3 which was initially only achieving SINR of $4.5978 \mathrm{~dB}$ but after the handover process, all users were able to achieve the SINR target of $5 \mathrm{~dB}$.

\subsubsection{Fixed-channel Based Treatment}

If the condition was impossible to move the channel or in single-channel condition, then it could use the method of DCPC [17] that made the power equal to maximum power $\left(P_{\max }\right)$ or GDCPC [18] that made the power equal to zero $\left(p_{i}^{*}=0\right)$, or using the proposed method HDCPC with a determined value which was a half of maximum power, $\frac{1}{2} P_{\max }$.

Table 1. Handover-channel based treatment

\begin{tabular}{|c|c|c|}
\hline \multirow{2}{*}{ User-Channel } & \multicolumn{2}{|c|}{ Channel Handover } \\
\cline { 2 - 3 } & SINR Before (dB) & SINR After (dB) \\
\hline User 1 Ch 1 & 5 & 5 \\
\hline User 1 Ch 3 & 4.5978 & 5 \\
\hline User 1 Ch 4 & 5 & 5 \\
\hline User 2 Ch 2 & 5 & 5 \\
\hline User 2 Ch 3 & 4.5978 & - \\
\hline User 2 Ch 4 & - & 5 \\
\hline User 3 Ch 1 & 5 & 5 \\
\hline User 3 Ch 2 & 5 & 5 \\
\hline User 3 Ch 3 & 4.5978 & 5 \\
\hline
\end{tabular}

Table 2. Fixed-channel based treatment

\begin{tabular}{|c|c|c|c|c|}
\hline \multirow{2}{*}{$\begin{array}{c}\text { User- } \\
\text { Channel }\end{array}$} & \multirow{2}{*}{$\begin{array}{c}\text { SINR } \\
\text { Before } \\
(\mathbf{d B})\end{array}$} & DCPC [17] & GDCPC [18] & \multirow{2}{*}{ HDCPC } \\
\cline { 3 - 5 } & 5 & 5 & 5 & 5 \\
\hline User 1 Ch 1 & 5 & 4.09219 & 0 & 4.09219 \\
\hline User 1 Ch 3 & 4.5978 & 5 & 5 & 5 \\
\hline User 1 Ch 4 & 5 & 5 & 5 & 5 \\
\hline User 2 Ch 2 & 5 & 5 & 5 & 5 \\
\hline User 2 Ch 3 & 4.5978 & 5 & 5 & 5 \\
\hline User 3 Ch 1 & 5 & 5 & 5 & 5 \\
\hline User 3 Ch 2 & 5 & 5 & 4.99998 & 5 \\
\hline User 3 Ch 3 & 4.5978 & 5 & & \\
\hline
\end{tabular}

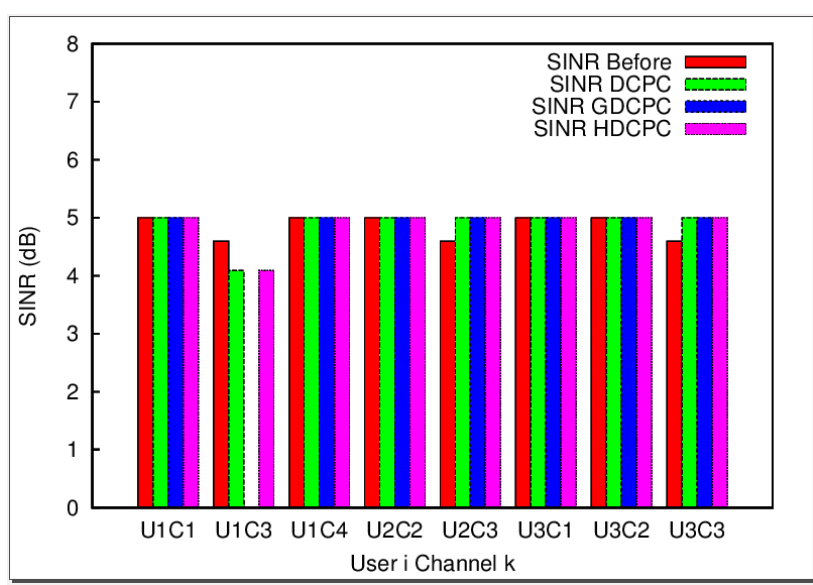

Figure.5 SINR Comparison in fixed-channel based

Table 2 showed the power usage on the HDCPC $\frac{1}{2} P_{\text {max }}$ that produced the same SINR when the user used a maximum power, $P_{\max }$. Based on these results, it can be concluded that the maximum power usage in DCPC method did not have much effect on the increasing of user SINR. This was because the user was already in the saturated conditions as the effect from the use of the channel at the same time or because of the influence of the distance between users that affecting the interference (the near-far effect). Thus, SINR resulting from use of $P_{\max }$ and $\frac{1}{2} P_{\max }$ was not much different or even the same, only the speed of iteration that distinguish the two methods. In addition, the use of methods of GDCPC made the user stops transmitting on the channel if the power exceeded the maximum power, $P_{\max }$, resulting in a better performance to other users and achieved the SINR target, but in reality it could be difficult to be implemented. Because the user had a power exceeding the maximum power, $P_{\max }$ was forced not to transmit.

In general, HDCPC is better than DCPC, because the HDCPC requires less power to get the same SINR using maximum power at DCPC. HDCPC is also more reasonable in implementation than GDCPC, because it is impossible to force the user not to transmit the power (at GDCPC) for the sake of another user, considering the character of the user who selfish in transmit-power usage. 
Figure 5 showed that user $i$ who used the channel $k$ is abbreviated by UiCk. It showed the comparison of the three methods: DCPC [17], GDCPC [18] and proposed HDCPC. From this figure it can be found that for a use using channel 3 (UiC3) had the initial SINR (SINR Before) less than the SINR target. After treatment, both at DCPC and HDCPC, the results of user 2 (U2C3) and user 3 (U3C3) were able to achieve the target SINR but user 1 (U1C3) could not achieve the SINR target. This was because user 1 is the farthest user from the FAP, so the signal became weaker and interference from other users would be higher. In addition, because the user 1 using a channel that is crowded, so the user needs greater power and cause interference to other users. This makes another user also increased power to maintain the SINR. This condition makes the users who use channel 3 are unable to achieve the SINR target.

\subsection{Ideal Feasible Condition}

Figure 6 showed the ideal feasible condition and convergent condition. It is said to be ideal because all users could converge to the value of power still below the maximum power, $P_{\max }$, making it eligible for feasibility and convergence. Low power usage occurred to user 3 either on the use of channel 1, channel 2 or channel 3. This was because this user had the closest distance with the FAP, so it did not require a high power to be able to transmit data.

Differences power value required by the user was caused due to the distance between the user and the amount of interference due to the use of channels simultaneously. Both of these factors were very influential, but the most significant influence was interference by the simultaneous use of channel.

Figure 6 showed that two users who consumed the most power were those using channel 3 , the channel most widely used simultaneously than any other channel.

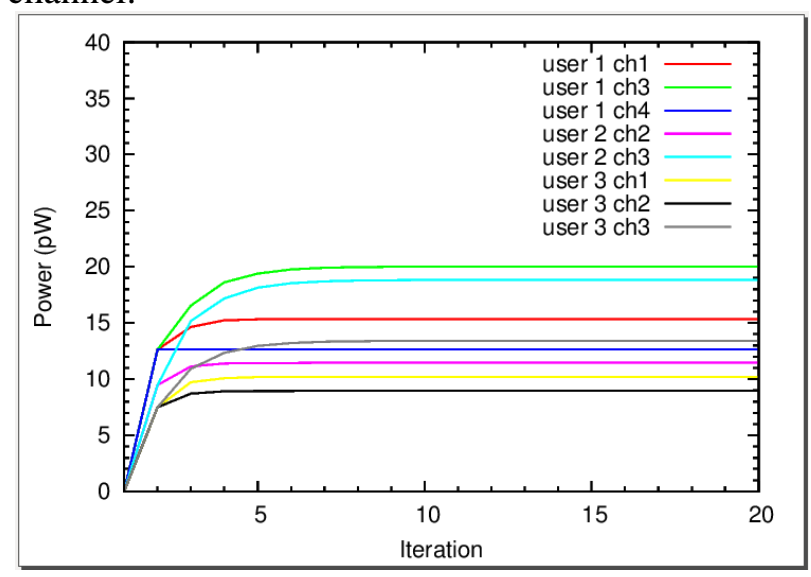

Figure.6 Power update in ideal feasible condition

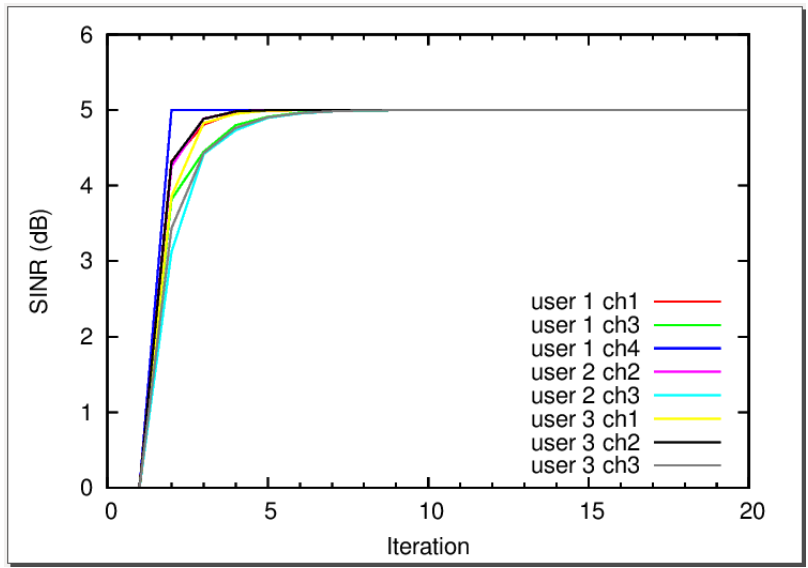

Figure.7 SINR user in ideal feasible condition

Figure 7 showed the user SINR during the iteration process in ideal feasible conditions. From this figure, it can be seen that all users were feasible and could achieve the conditions converges the SINR target value which was equal to $5 \mathrm{~dB}$.

\subsection{Analysis of Convergence}

This analysis was based on the test on the speed of convergence (convergence rate) at DPC with some ideal conditions by testing two SINR target values. This analysis was not performed on Sooyeol [21] because there are not applying the iteration method for power update process, so that the convergence of the system cannot be analyzed. In this study, the given SINR targets were $5 \mathrm{~dB}$ and $9.9 \mathrm{~dB}$ used to test the convergence of the system.

As seen in Table 3, in SINR target of $5 \mathrm{~dB}$, both the power and SINR had fast iteration within less than 10 iterations to achieve the convergence. While at time the SINR target increased to $9.9 \mathrm{~dB}$, the iteration became slower in achieving convergence, in the range of 25 iterations for the channel with more users. It can be concluded that the larger the SINR target, the longer iterations required to achieve convergent condition. In other words, the convergence rate is inversely proportional to the SINR target. In addition, related to the analysis of the convergence speed, the more the number of users simultaneously using the channel, the longer the number of iterations required to reach the convergent condition, and vice versa.

Table 3. Convergence rate of power and SINR

\begin{tabular}{|c|c|c|c|c|}
\hline \multirow[b]{2}{*}{$\begin{array}{l}\text { User- } \\
\text { channel }\end{array}$} & \multicolumn{2}{|c|}{ SINR Target of $5 \mathrm{~dB}$} & \multicolumn{2}{|c|}{ SINR Target of $9.9 \mathrm{~dB}$} \\
\hline & $\begin{array}{c}\text { Power } \\
\text { Iteration }\end{array}$ & $\begin{array}{c}\text { SINR } \\
\text { Iteration }\end{array}$ & $\begin{array}{c}\text { Power } \\
\text { Iteration }\end{array}$ & $\begin{array}{c}\text { SINR } \\
\text { Iteration }\end{array}$ \\
\hline User $1 \mathrm{Ch} 1$ & 6 & 6 & 12 & 10 \\
\hline User $1 \mathrm{Ch} 3$ & 9 & 8 & 28 & 24 \\
\hline User $1 \mathrm{Ch} 4$ & 2 & 2 & 2 & 2 \\
\hline User $2 \mathrm{Ch} 2$ & 4 & 5 & 8 & 8 \\
\hline User 2 Ch 3 & 8 & 8 & 25 & 25 \\
\hline User $3 \mathrm{Ch} 1$ & 5 & 6 & 9 & 10 \\
\hline User $3 \mathrm{Ch} 2$ & 5 & 5 & 8 & 8 \\
\hline User $3 \mathrm{Ch} 3$ & 9 & 8 & 27 & 25 \\
\hline
\end{tabular}


From Table 3, it can be concluded that besides affect to the feasibility, multi-channel and channel sharing usage are also greatly affect to the convergence rate.

From Figures 8 and 9, it can be shown that the user using the channel 3 (U1C3,U2C3 and $U 3 C 3$ ) required at most iterations to achieve convergent than users using other channels, while the user using channel 4 required the less iterations. It can be concluded that the more user using the same channel, the more iterations required for achieving convergence. In other words, convergence rate was inversely proportional to the number of users per channel.

In addition, as shown in Fig. 8 and 9 can also be seen that the user 1 channel 4 (U1C4) had the fast convergence rate equal to the both of SINR target, namely 2 iterations. This means that changes to the SINR target did not affect the user using the channel itself (single user for single channel). This was because interference that received by the user was very little.

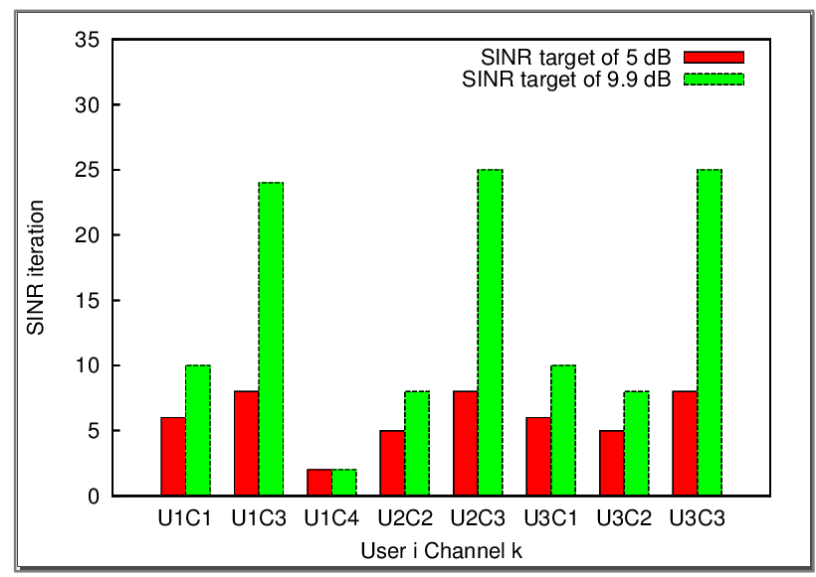

Figure. 8 Convergence rate of SINR iteration when SINR target is changed

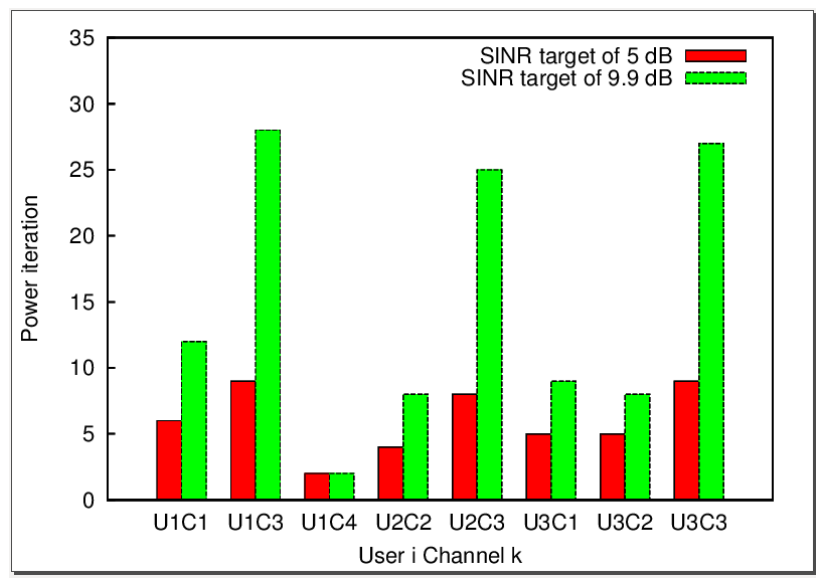

Figure.9 Convergence rate of power iteration when SINR target is changed

\section{Conclusion}

Based on the results, it can be concluded that when the results of eigenvalue of matrix $\boldsymbol{H}$ was less than 1 so as to produce a non-negative power vector means that the system was feasible. When the system is ideal feasible then all users will be able to achieve the SINR target and the power will be convergent in a unique point that less than maximum power. But when the system is semi feasible then some users will not be able to achieve the SINR target and the power will be convergent in a unique point that more than maximum power.

The use of channels in a simultaneous manner by many users will influence the feasibility and convergence. The more users who use the channel, feasibility and convergence will be difficult to be achieved. Similar with distance, the farther the distance of user to the FAP and the closer distance of the user to the interferer could lead to, the greater interference making the feasibility and convergence increasingly difficult to be achieved.

From the DPC analysis it can be concluded that the maximum power usage that did not have much effect on the increasing of user SINR. This was because the user was already in the saturated conditions as the effect from the use of the channel at the same time or because of the influence of the distance between users affecting the interference (the near-far effect). Thus, the use of HDCPC will have an effect on the efficiency of power usage when it compared with DCPC, and HDCPC would be more implementable compared to GDCPC. In addition, related to the convergence analysis, it can be concluded that the larger the SINR target, the longer iterations required to achieve convergent condition. In other words, the convergence rate is inversely proportional to the SINR target.

Future work of distributed power control is novel Power Control Game (PCG), which is power control based on Game Theory in cognitive femtocell network. This method is expected to have less power, higher in achieving SINR and better in convergence rate.

\section{Acknowledgments}

This work was supported by the Department of Electrical Engineering and Information Technology, Faculty of Engineering, Universitas Gadjah Mada, Yogyakarta, Indonesia.

\section{References}

[1] S. A. Rabee, B. S. Sharif, and S. Sali, "An efficient algorithm for distributed power 
control in cellular radio systems," $3 G$ Mobile Communication Technologies, Third International Conference on (Conf. Publ. No. 489), pp. 123-127, 2002.

[2] J. Zander, "Performance of optimum transmitter power control in cellular radio systems," Veh. Technol. IEEE Trans. On, Vol. 41, No. 1, pp. 57-62, 1992.

[3] A. F. Isnawati, R. Hidayat, S. Sulistyo, and I. W. Mustika, "A comparative study on centralized and distributed power control in cognitive femtocell network," 8th International Conference on Information Technology and Electrical Engineering (ICITEE), 2016.

[4] N. Chakchouk and B. Hamdaoui, "QoS-aware autonomous distributed power control in cochannel femtocell networks," Global Communications Conference (GLOBECOM), IEEE, pp. 567-571, 2012.

[5] J. Duan, J. Liu, S. Leng, and Q. Wang, "A game-based power control scheme for cognitive radio networks," Computational Problem-Solving (ICCP), International Conference on, pp. 76-79, 2012.

[6] Z. Lu, Y. Sun, X. Wen, T. Su, and D. Ling, "An energy-efficient power control algorithm in femtocell networks," Computer Science \& Education (ICCSE), 7th International Conference on, pp. 395-400, 2012.

[7] A. J. Luah and C. K. Tan, "A Nash-based power control game for green communications via cognitive radio networks," Sustainable Utilization and Development in Engineering and Technology (STUDENT), IEEE Conference on, pp. 164-169, 2012.

[8] J. Jiao, L. Jiang, and C. He, "A novel game theoretic utility function for power control in cognitive radio networks," International Conference on Computational and Information Sciences, ICCIS, pp. 1553-1557, 2013.

[9] Z. Junhui, Y. Tao, G. Yi, W. Jiao, and F. Lei, "Power control algorithm of cognitive radio based on non-cooperative game theory," Commun. China, Vol. 10, No. 11, pp. 143-154, 2013.

[10] A. F. Isnawati, R. Hidayat, S. Sulistyo, and I. W. Mustika, "Feasible solution of centralized power control for multi-channel cognitive femtocell network," 7th International Conference on Information Technology and Electrical Engineering (ICITEE), 2015.

[11] M. Rasti, A. R. Sharafat, and J. Zander, "Pareto and energy-efficient distributed power control with feasibility check in wireless networks," IEEE Trans. Inf. Theory, Vol. 57, No. 1, pp. 245-255, Jan. 2011.

[12] M. Rasti, M. Hasan, L. B. Le, and E. Hossain, "Distributed uplink power control for multicell cognitive radio networks," IEEE Trans. Commun., Vol. 63, No. 3, pp. 628-642, Mar. 2015.

[13] L. Qian, X. Li, J. Attia, and Z. Gajic, "Power control for cognitive radio ad hoc networks," Local \& Metropolitan Area Networks, LANMAN, 15th IEEE Workshop on, pp. 7-12, 2007.

[14] N. Nie, C. Comaniciu, and P. Agrawal, "A game theoretic approach to interference management in cognitive networks," Wireless Communications, Springer, pp. 199-219, 2007.

[15] G. J. Foschini and Z. Miljanic, "A simple distributed autonomous power control algorithm and its convergence," IEEE Trans. Veh. Technol., Vol. 42, No. 4, pp. 641-646, Nov. 1993.

[16] S. Koskie and Z. Gajic, "A Nash game algorithm for SIR-based power control in 3G wireless CDMA networks," IEEEACM Trans. Netw., Vol. 13, No. 5, pp. 1017-1026, Oct. 2005.

[17] S. A. Grandhi and J. Zander, "Constrained power control in cellular radio systems," Vehicular Technology Conference, IEEE 44th, pp. 824-828, 1994.

[18] F. Berggren, R. Jäntti, and S.-L. Kim, “A generalized algorithm for constrained power control with capability of temporary removal," Veh. Technol. IEEE Trans. On, Vol. 50, No. 6, pp. 1604-1612, 2001.

[19] Y. A. Al-Gumaei, K. A. Noordin, A. W. Reza, and K. Dimyati, "A new power control game in two-tier femtocell networks," Telematics and Future Generation Networks (TAFGEN), 1st International Conference on, pp. 131-135, 2015.

[20] X. Li, L. Qian, and D. Kataria, "Downlink power control in co-channel macrocell femtocell overlay," Information Sciences and Systems, CISS. 43rd Annual Conference on, pp. 383-388, 2009.

[21] S. Im, H. Jeon, and H. Lee, "Autonomous distributed power control for cognitive radio networks," Vehicular Technology Conference, VTC, IEEE 68th, pp. 1-5, 2008.

[22] H. Koivo and M. Elmusrati, Systems engineering in wireless communication, Hoboken, NJ: John Wiley \& Sons, 2009. 
[23] V. Chandrasekhar, J. G. Andrews, T. Muharemovic, Z. Shen, and A. Gatherer, "Power control in two-tier femtocell networks," IEEE Trans. Wirel. Commun., Vol. 8, No. 8, pp. 4316-4328, Aug. 2009.

[24] O. Durowoju, K. Arshad, and K. Moessner, "Distributed power control algorithm for cognitive radios with primary protection via spectrum sensing under user mobility," $A d$ Hoc Netw., Vol. 10, No. 5, pp. 740-751, Jul. 2012.

[25] R. D. Yates, "A framework for uplink power control in cellular radio systems," Sel. Areas Commun. IEEE J. On, Vol. 13, No. 7, pp. 1341-1347, 1995.

[26] A. Al Talabani, A. Nallanathan, and H. X. Nguyen, "A novel chaos based cost function for power control of cognitive radio networks," IEEE Commun. Lett., Vol. 19, No. 4, pp. 657660, Apr. 2015.

[27] T. Holliday, N. Bambos, P. Glynn, and A. Goldsmith, "Distributed power control for time varying wireless networks optimality and convergence," Proceedings of the annual ALLERTON Conference on Communication Control and Computing, 2012.

[28] L. I. Dong, D. A. I. Xianhua, and H. Zhang, "Game theoretic analysis of joint rate and power allocation in cognitive radio networks," Int. J. Commun. Netw. Syst. Sci., Vol. 2, No. 01, 2009. 